\title{
Facet Cooling in High-Power InGaAs/AlGaAs Lasers
}

\author{
Seval Arslan ${ }^{(}$, Sinan Gündoğdu${ }^{\circledR}$, Abdullah Demir, and Atilla Aydınlı
}

\begin{abstract}
Several factors limit the reliable output power of a semiconductor laser under CW operation, such as carrier leakage, thermal effects, and catastrophic optical mirror damage (COMD). Ever higher operating powers may be possible if the COMD can be avoided. Despite exotic facet engineering and progress in non-absorbing mirrors, the temperature rise at the facets puts a strain on the long-term reliability of these diodes. Although thermoelectrically isolating the heat source away from the facets with non-injected windows helps lower the facet temperature, data suggests the farther the heat source is from the facets, the lower the temperature. In this letter, we show that longer non-injected sections lead to cooler windows and biasing this section to transparency eliminates the optical loss. We report on the facet temperature reduction that reaches below the bulk temperature in high power InGaAs/AlGaAs lasers under QCW operation with electrically isolated and biased windows. Acting as transparent optical interconnects, biased sections connect the active cavity to the facets. This approach can be applied to a wide range of semiconductor lasers to improve device reliability as well as enabling the monolithic integration of lasers in photonic integrated circuits.
\end{abstract}

Index Terms-Semiconductor lasers, diode lasers, high power lasers, catastrophic optical damage, reliability.

\section{INTRODUCTION}

D ESPITE record-high output powers [1] and electrooptical power conversion efficiencies [2], catastrophic optical damage (COD) has been known and recognized as one of the limitations of semiconductor lasers since their invention [3]. This type of heating induced device failure is a key to both device performance and reliability [4]. Many successful methods were reported in the literature to prevent facet mirror reaching the critical temperature of $\mathrm{T}_{c}=120$ $160{ }^{\circ} \mathrm{C}$ and causing COD at the output mirror facet (COMD) [3]. Non-absorbing mirrors (NAMs) close to the facets and/or non-injecting current windows have been implemented to reduce optical absorption and facet heating [5], [6]. NAMs are produced by quantum well-intermixing close to the mirrors [2]. However, this method is a complex process with tight fabrication tolerances [7]. In non-injected current blocking

Manuscript received August 14, 2018; revised November 13, 2018; accepted November 26, 2018. Date of publication November 30, 2018; date of current version December 24, 2018. This work was supported by Ermaksan A.S. (Corresponding author: Atilla Aydinlı.)

S. Arslan and S. Gündoğdu are with the Physics Department, Bilkent University, 06800 Ankara, Turkey (e-mail: sevalsaritas67@gmail.com).

A. Demir is with the UNAM-National Nanotechnology Research Center, Institute of Materials Science and Nanotechnology, Bilkent University, 06800 Ankara, Turkey (e-mail: abdullah.demir@unam.bilkent.edu.tr.)

A. Aydinlı is with the Electrical and Electronics Engineering Department, Uludağ University, 16059 Bursa, Turkey.

Color versions of one or more of the figures in this letter are available online at http://ieeexplore.ieee.org.

Digital Object Identifier 10.1109/LPT.2018.2884465 window approach, the facet is separated from the current injection window by placing the pump electrode away from the facet. Facet temperature was shown to be lower using a $30 \mu \mathrm{m}$ compared to $0 \mu \mathrm{m}$ long current blocking region due to reduced current density near facet [8]. However, the length of the unpumped current blocking window is limited due to the optical absorption in this region degrading the quantum efficiency of the lasers [9]. Alternatively, ultra-high vacuum cleaving and passivation of the facets eliminates the surface states and leads to higher COMD thresholds at the cost of higher complexity and expense of fabrication [10]-[12]. So far, these approaches have achieved effective results increasing $\mathrm{T}_{c}$. However, none of these studies have demonstrated laser facet temperature lower than its bulk or cavity. Although eliminating the surface recombination and optical absorption is critical for the reduction of mirror temperature as targeted in the previous methods, self-heat load contributes greatly and is the dominant source of facet heating, but it has not been addressed before, which is targeted by the method reported in this letter.

In this work, facet temperature is reduced using a new, biased window approach in InGaAs/AlGaAs high power laser diodes without degrading output power. To reduce the facet temperature, the laser chip is monolithically separated into two regions by dividing the top electrode to lasing and transparent window sections. This configuration allows keeping the high self-heat load of the lasing region away from the heat sensitive output facet. The transparent window, next to the facet, operating at very low current produces negligible heat. We used thermoreflectance spectroscopy with a resolution below $1 \mathrm{~K}$ [2], [3], [13]-[18]. The temperature distribution on the facet under operating current is determined by a full-scale image of the object plane. The facet temperature of a $5.00 \mathrm{~mm}$ long laser was compared with lasers of the same length that employed $0.75 \mathrm{~mm}$ and $1.00 \mathrm{~mm}$ long biased windows. The output power of the laser with biased windows while at transparency was brought to the same level as the output power of the control lasers. Facet temperature was observed to decrease by $40 \%$ in the waveguide region. We note that the reduced facet temperatures are also below the bulk temperature of the $5.00 \mathrm{~mm}$ long control laser demonstrating a cooling effect on laser facet. Reduction of large facet temperature is by $30 \%$ below the bulk temperature of the control laser. Our results indicate that biased window approach should lead to increased COMD threshold level and reliability of the semiconductor lasers.

\section{EXPERIMENTAL}

The layer structure of the GaAs-based high-power laser emitting around $915 \mathrm{~nm}$ is composed of an n-GaAs sub- 
strate, $3000 \mathrm{~nm} \mathrm{n}$-AlGaAs cladding, $500 \mathrm{~nm} \mathrm{n}$-AlGaAs waveguide, $8 \mathrm{~nm}$ InGaAs QW, $500 \mathrm{~nm}$ p-AlGaAs waveguide, $1000 \mathrm{~nm}$ p-AlGaAs cladding and finally $100 \mathrm{~nm}$ GaAs cap layer [19]. In this study, $100 \mu \mathrm{m}$ wide broad area high power laser diodes with different lasing cavity and window lengths were used to compare the performance of the lasers and their facet temperatures. The main difference between the designs under investigation is the presence of an electrically isolated window region. The control lasers have cavity lengths of 4.00 and $5.00 \mathrm{~mm}$, whereas coupled cavities are $4.25+0.75 \mathrm{~mm}$ and $4.00+1.00 \mathrm{~mm}$, where 0.75 and $1.00 \mathrm{~mm}$ are the window lengths. Ridge waveguides were formed on the same wafer by wet etching, a dielectric layer $\left(\mathrm{Si}_{3} \mathrm{~N}_{4}\right)$ was deposited as the electrical insulator, and p-contact windows were opened on top of the waveguide regions. $\mathrm{Ti} / \mathrm{Pt} / \mathrm{Au}$ and $\mathrm{AuGe} / \mathrm{Ni} / \mathrm{Au}$ were deposited for $\mathrm{p}$ - and $\mathrm{n}$ - metals, respectively, and alloyed to obtain ohmic contacts. $300 \mathrm{~nm}$ deep trenches for electrical isolation between the laser and window regions were opened after the formation of the waveguide. Trench depth was limited to $300 \mathrm{~nm}$ to conserve the mode profile and not to introduce optical losses. The resistance between the laser and window regions was measured as $69 \Omega$. Fabricated lasers were cleaved and mounted epi-up on a copper block.

Facet temperatures of the diode lasers were measured by CMOS based-thermoreflectance method using the 'fourbucket' technique [10]. For thermoreflectance measurements, a LED at $450 \mathrm{~nm}$ is used as the probe light and directed onto the laser facet through a 0.40 numerical aperture $20 \times$ microscope objective. Lasers were mounted on a copper baseplate for testing at room temperature and they are tested up to $5 \mathrm{~A}$ injection current $\left(\mathrm{I}_{1}\right)$. They were biased by LDX-3600 diode driver under QCW current injection with $20 \mathrm{~ms}$ pulse duration and $20 \%$ duty cycle. Lasers with biased windows were tested under various window bias currents $\left(I_{2}\right)$ of $0,50,100,150$, $170 \mathrm{~mA}$ while lasing with current $\mathrm{I}_{1}$. The temperature modulated back-reflected light is collected with CMOS camera triggered by a diode driver and analyzed by a computer. The relation between the relative change in reflectivity and the temperature change is given as [10], [14], [20].

$$
\frac{\Delta R}{R}=\left(\frac{1}{R} \frac{\partial R}{\partial T}\right) \Delta T=\kappa \Delta T
$$

where $\kappa$, is the thermoreflectance coefficient that depends on the sample material and probe light wavelength [10]. We calculated the thermoreflectance constant of GaAs substrate at $450 \mathrm{~nm}$ as $(3.2 \pm 0.6) \times 10^{-4}$ by modulating the laser temperature using a Newport 3700 temperature controller. Since the aim is to compare facet temperature changes between a $5.00 \mathrm{~mm}$ long high-power laser with 0.75 and $1.00 \mathrm{~mm}$ long biased windows and a $5.00 \mathrm{~mm}$ long control laser, possible differences between $\kappa$ 's of GaAs and AlGaAs layers that make up the laser are not critical. Temperature resolution during the measurements was $\pm 0.1^{\circ} \mathrm{C}$ with a spatial resolution of $\pm 0.7 \mu \mathrm{m}$.

\section{RESULTS AND DISCUSSION}

All samples were characterized by measuring L-I-V curves under $\mathrm{CW}$ for each biased window current $\left(\mathrm{I}_{2}\right)$ and

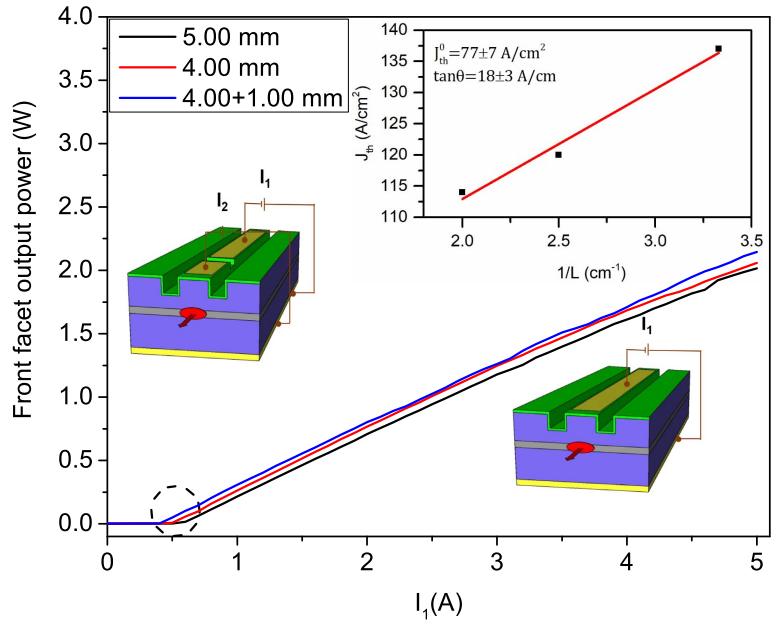

Fig. 1. Front facet output power at various injection currents for standard $4.00 \mathrm{~mm}$ and $5.00 \mathrm{~mm}$ long lasers and $5.00 \mathrm{~mm}$ long laser with $1.00 \mathrm{~mm}$ long window region (window current is at $170 \mathrm{~mA}$ ). The schematic illustration of the two different laser designs is also shown. Inset shows the threshold current density versus inverse cavity length line graph.

threshold currents of the lasers were determined for each bias current. To illustrate the performance of the biased window, we compare the L-I characteristics of the $5.00 \mathrm{~mm}$ long laser that has a $1.00 \mathrm{~mm}$ window section biased at $\mathrm{I}_{2}=$ $170 \mathrm{~mA}$, with standard 4.00 and $5.00 \mathrm{~mm}$ lasers, in Fig. 1. In this study, we characterized uncoated and epi-up lasers to simplify the observation of the facet cooling. The power conversion efficiency of the lasers is around 52\%, which is mainly limited by uncoated and epi-up mounted operation. The threshold currents of the $4.00 \mathrm{~mm}, 5.00 \mathrm{~mm}$ long lasers and that of the $4.00+1.00 \mathrm{~mm}$ long laser biased at $\mathrm{I}_{2}=$ $170 \mathrm{~mA}$, are $482 \mathrm{~mA}, 568 \mathrm{~mA}$ and $408 \mathrm{~mA}$, respectively. We observe that the threshold current of the laser decreases as a function of window bias as the biased window section of the structure is brought to transparency compensating for the loss. Increasing the bias current increases the spontaneous emission, hence contributing to the gain in the pump section. Therefore, the threshold current of the $4.00+1.00 \mathrm{~mm}$ long laser is smaller than that of the $4.00 \mathrm{~mm}$ long conventional laser for $170 \mathrm{~mA}$ bias current. Furthermore, considering that threshold current is an exponential function of temperature, the colder laser $(4.00+1.00 \mathrm{~mm}$ long $)$ has a lower threshold current. We measured the optical power at different window bias currents. The output power of the 4.00 and $5.00 \mathrm{~mm}$ long lasers and that of the laser with window biased at $170 \mathrm{~mA}$ are $2.06 \mathrm{~W}, 2.15 \mathrm{~W}$ and $2.02 \mathrm{~W}$ at $5 \mathrm{~A}$ pump current, respectively. The transparency and threshold currents of the window are calculated using the basic laser rate equations [21]. Threshold current density in the limit of infinite cavity length, $J_{t h}^{0}$, and $\tan \theta$ are found from the intercept and slope of the threshold current density versus inverse cavity length line graph (inset of Fig.1), respectively, as $77 \pm 7 \mathrm{~A} / \mathrm{cm}^{2}$ and $18 \pm 3 \mathrm{~A} / \mathrm{cm}$, the internal loss, $\alpha_{i}$, is calculated as $0.39 \mathrm{~cm}^{-1}$ (not shown). Hence, the transparency and threshold currents for $1.00 \mathrm{~mm}$ long window are estimated as $76 \pm 7 \mathrm{~mA}$ (indicated in Fig.3) and $257 \pm 28 \mathrm{~mA}$, respectively. 

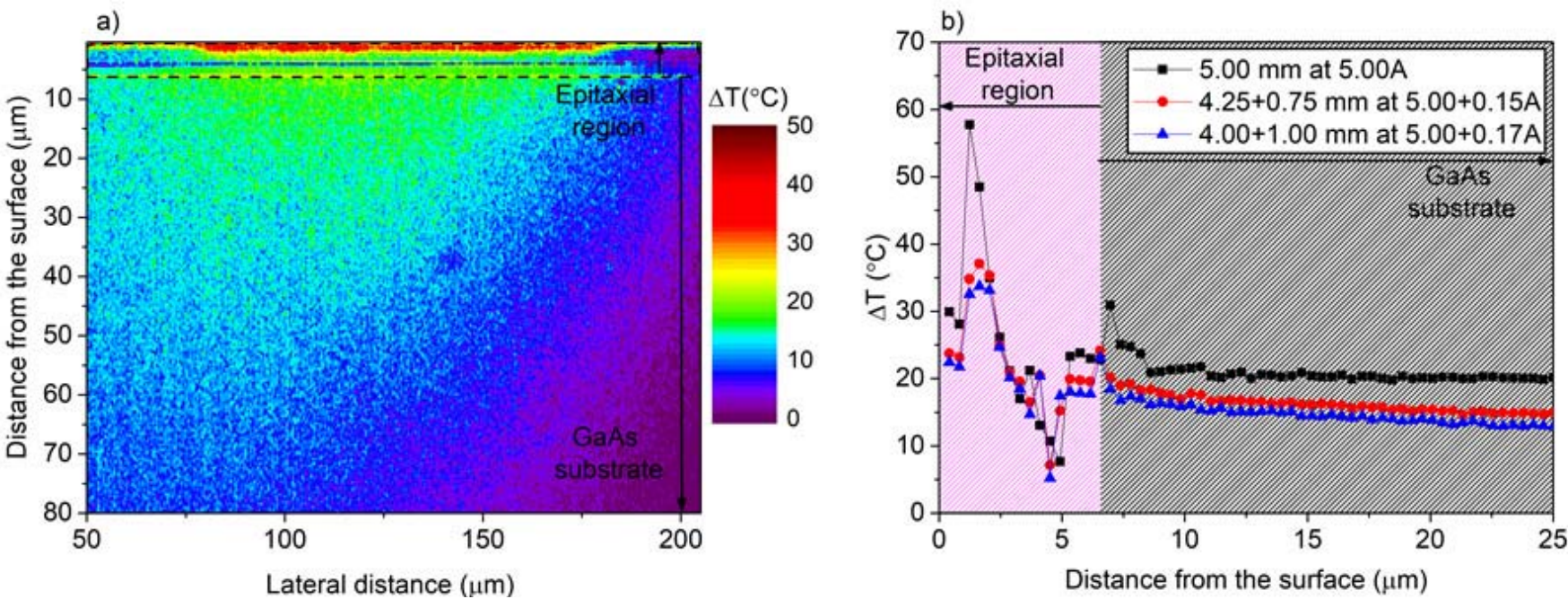

Fig. 2. (a) Temperature map of $4.00+1.00 \mathrm{~mm}$ long laser at $\mathrm{I}_{1}=5.00 \mathrm{~A}$ and $\mathrm{I}_{2}=0.17 \mathrm{~A}$ and (b) Temperature line scans for $5.00 \mathrm{~mm}, 4.25+0.75 \mathrm{~mm}$ and $4.00+1.00 \mathrm{~mm}$ long lasers taken at the active region through the direction perpendicular to surface.

Fig.2 shows the facet temperature measurement results at $\mathrm{I}_{1}=5$ A for $5.00 \mathrm{~mm}, 4.25+0.75 \mathrm{~mm}$ (with $\mathrm{I}_{2}=150 \mathrm{~mA}$ ) and $4.00+1.00 \mathrm{~mm}$ (with $\mathrm{I}_{2}=170 \mathrm{~mA}$ ) lasers. Fig. 2 a shows the temperature distribution map of $4.00 \mathrm{~mm}+1.00 \mathrm{~mm}$ laser and Fig. 2b shows vertical temperature scans (from the top of the epitaxial region to GaAs substrate) for $5.00 \mathrm{~mm}$, $4.25+0.75 \mathrm{~mm}$ and $4.00+1.00 \mathrm{~mm}$ long lasers, respectively. As seen in Fig. 2a, active region has the highest temperature and heat spreads vertically towards the copper heat sink as well as laterally through the electroplated gold heat spreader. In Fig. 2a, we note that the right-hand side of the mesa is cooler than the left-hand side due to its larger spacing from the edge of the chip compared to that of the left. In Fig. 2b, temperature rise along the facet vertical from the top of the epitaxial region to GaAs substrate is plotted as a function of distance from the epitaxial surface. The average temperature is determined along the $100 \mu \mathrm{m}$ long width of the ridge facet. For $4.00+1.00 \mathrm{~mm}$ long lasers, facet temperature fluctuations $\left(\mathrm{T}_{a v}=54 \pm 0.4^{\circ} \mathrm{C}\right)$ are nearly homogenous while $5.00 \mathrm{~mm}$ long lasers show clear filamentation $\left(\mathrm{T}_{a v}=79 \pm 3.0{ }^{\circ} \mathrm{C}\right)$ (not shown). In fact, the temperature inside the filaments in $5.00 \mathrm{~mm}$ long lasers reaches up to $150{ }^{\circ} \mathrm{C}$ while the temperature in the $4.00+1.00 \mathrm{~mm}$ long laser rises only up to $65{ }^{\circ} \mathrm{C}$ at most. Both the magnitude of the temperature fluctuations as well as the comparison of the hottest regions show that $4.00+1.00 \mathrm{~mm}$ long biased window lasers operating at similar optical output power have much cooler facet than that of the $5.00 \mathrm{~mm}$ long control lasers. One can also safely conclude that biased window approach increases the threshold for filament formation. The waveguide region has the highest temperature and the temperature falls towards the GaAs substrate as seen in the temperature map. Sharp changes in the vertical scans are mainly due to the differences in the reflectivities of materials that make up the epitaxial layer, and consequently are indicative of different values of thermoreflectance. Thus, sharp peaks delineate the transition between the growth layers (e.g., the peaks on the GaAs substrate side in Fig. 2b, are indicative of the epitaxial growth interface). As this is a comparative study, and all layers are the same in all samples, changes in facet temperature for $4.00+1.00 \mathrm{~mm}$ and $4.25+0.75 \mathrm{~mm}$ long lasers at waveguide region with respect to that of $5.00 \mathrm{~mm}$ long laser is not expected to be significantly affected assuming the same constant for all layers that of $\mathrm{GaAs}$ as (i. e. $\left.(3.2 \pm 0.6) \times 10^{-4}\right)$. The spatial resolution of $\pm 0.7 \mu \mathrm{m}$ allows us to characterize the temperature changes in the waveguide region including the active region. We find that the facet temperature of the waveguide region in $4.00+1.00 \mathrm{~mm}$ laser is reduced by $40 \%$ demonstrating that a biased window at transparency leads to cooler windows.

In Fig.3, we compare the temperature rise and front facet output power of the $5.00 \mathrm{~mm}$ long conventional laser and $4.00+1.00 \mathrm{~mm}$ long lasers as a function of window bias current $\left(\mathrm{I}_{2}\right)$ at different pump currents $\left(\mathrm{I}_{1}\right)$. As expected, facet temperature increases with increasing $\mathrm{I}_{1}$. The thermal resistance of $5.00 \mathrm{~mm}$ long laser is determined as $12.8 \mathrm{~K} / \mathrm{W}$ using the wavelength shift of Fabry-Perot modes. Hence, the bulk temperatures of $5.00 \mathrm{~mm}$ long laser at $\mathrm{I}_{1}$ of $1 \mathrm{~A}, 3 \mathrm{~A}$ and $5 \mathrm{~A}$ are $12.4,27.8$, and $49.7{ }^{\circ} \mathrm{C}$, respectively. The facet temperatures of this laser at the waveguide region for the same currents were measured as $14.7,33.7$, and $58.7{ }^{\circ} \mathrm{C}$, respectively. The facet temperatures are higher than the bulk temperatures as expected, due to the non-radiative recombination at the laser facet. We measured the facet temperatures of $4.00+1.00 \mathrm{~mm}$ long lasers at the waveguide region as a function of $I_{2}$ for different $I_{1}$ values. For higher $I_{1}$, the temperature of the $4.00+1.00 \mathrm{~mm}$ long laser increases correlated with dissipated power. There is a slight temperature increase at window bias current of $\mathrm{I}_{2}=50 \mathrm{~mA}$. This is due to Joule heating of the window since the window is not transparent at this bias current. $\mathrm{I}_{2}=50 \mathrm{~mA}$ is below the calculated window transparency current level of $76 \mathrm{~mA}$ and thus generates $69 \mathrm{~mW}$ of dissipated power corresponding to $0.9{ }^{\circ} \mathrm{C}$ temperature rise in agreement with the experimental data. Temperature decreases slightly after reaching $\mathrm{I}_{2}=100 \mathrm{~mA}$, which is above the transparency 


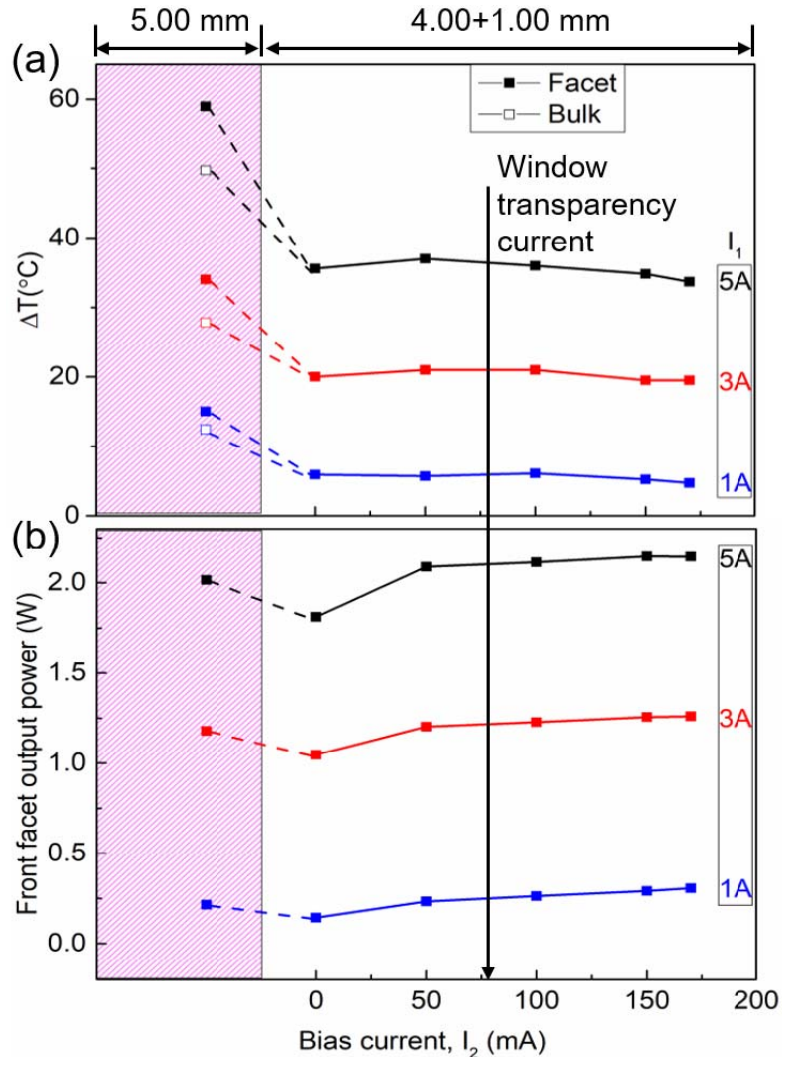

Fig. 3. Comparing $5.00 \mathrm{~mm}$ and $4.00+1.00 \mathrm{~mm}$ long lasers at various laser currents as a function of window bias current: (a) Change in facet and bulk temperatures (b) Front facet output power.

current and hence optical absorption losses are eliminated. The temperature is reduced by $40 \%$ at the facet and $30 \%$ compared to the bulk using $1.00 \mathrm{~mm}$ long window at $5 \mathrm{~A}$ injection current with $170 \mathrm{~mA}$ window bias current. Fig. $3 \mathrm{~b}$ shows that the transparent window and cooler facet result at higher laser powers for all pump currents. The front facet output powers of the $5.00 \mathrm{~mm}$ long laser at various currents of $1 \mathrm{~A}, 3 \mathrm{~A}$ and $5 \mathrm{~A}$ are $0.22,1.18$ and $2.02 \mathrm{~W}$, respectively. The front facet output power of the laser with a $1.00 \mathrm{~mm}$ long window region is reduced by $10 \%$ compared to the $5.00 \mathrm{~mm}$ long laser when the window region is not pumped $\left(\mathrm{I}_{2}=0 \mathrm{~mA}\right)$. However, its output recovers and reaches the same power level of $5.00 \mathrm{~mm}$ long laser when the window is biased above the transparency current.

\section{SUMMARY}

In summary, we demonstrate a promising method of facet temperature reduction for semiconductor lasers achieving facet temperatures far below the bulk temperature of the laser cavity without compromising the laser performance. This approach can be considered as a monolithic cavity consisting of laser and window sections with the latter biased to transparency. The facet temperature of a high-power diode laser was reduced by $40 \%$ in the waveguide region when a $1.00 \mathrm{~mm}$ long transparent window is used. The output powers of the lasers with and without windows are comparable. Open to further optimization, this novel idea of electrically biased transparent sections integrated with a laser cavity can be applied in laser bars and in DFB laser integrating monolithic photonics circuits to improve their performance and long-term reliability.

\section{REFERENCES}

[1] A. Demir, M. Peters, R. Duesterberg, V. Rossin, and E. Zucker, "Semiconductor laser power enhancement by control of gain and power profiles," IEEE Photon. Technol. Lett., vol. 27, no. 20, pp. 2178-2181, Oct. 15, 2015.

[2] A. Knigge et al., "Passively cooled $940 \mathrm{~nm}$ laser bars with $73 \%$ wallplug efficiency at $70 \mathrm{~W}$ and $25^{\circ} \mathrm{C}$," Electron. Lett., vol. 41, no. 5, pp. 250-251, Mar. 2005.

[3] J. W. Tomm, M. Ziegler, M. Hempel, and T. Elsaesser, "Mechanisms and fast kinetics of the catastrophic optical damage (COD) in GaAs-based diode lasers," Laser Photon. Rev., vol. 5, no. 3, pp. 422-441, Mar. 2011.

[4] M. Ziegler et al., "Physical limits of semiconductor laser operation: A time-resolved analysis of catastrophic optical damage," Appl. Phys. Lett., vol. 97, no. 2, p. 021110, Jul. 2010.

[5] P. G. Piva et al., "Reduction of InGaAs/GaAs laser facet temperatures by band gap shifted extended cavities," Appl. Phys. Lett., vol. 70, no. 13, pp. 1662-1664, Mar. 1997.

[6] J. Michaud et al., "Precise facet temperature distribution of high-power laser diodes: Unpumped window effect," IEEE Photon. Technol. Lett., vol. 27, no. 9, pp. 1002-1005, May 1, 2015.

[7] P. W. Epperlein, Basic Diode Laser Engineering Principles. Hoboken, NJ, USA: Wiley, 2013.

[8] F. Rinner et al., "Facet temperature reduction by a current blocking layer at the front facets of high-power InGaAs/AlGaAs lasers," J. Appl. Phys., vol. 93, no. 3, pp. 1848-1850, 2003.

[9] J. Hendrix, G. Morthier, and R. Baets, "Influence of laser parameters and unpumped regions near the facets on the power level for catastrophic optical damage in short wavelength lasers," IEE Proc.-Optoelectron., vol. 144, no. 2, pp. 109-114, Apr. 1997.

[10] L. W. Tu, E. F. Schubert, M. Hong, and G. J. Zydzik, "In-vacuum cleaving and coating of semiconductor laser facets using thin silicon and a dielectric," J. Appl. Phys., vol. 80, no. 11, pp. 6448-6451, Dec. 1996.

[11] N. Chand, W. S. Hobson, J. F. de Jong, P. Parayanthal, and U. K. Chakrabarti, "ZnSe for mirror passivation of high power GaAs based lasers," Electron. Lett., vol. 32, no. 17, pp. 1595-1596, Aug. 1996.

[12] P. Ressel, G. Erbert, G. Beister, C. Dzionk, and G. Trankle, "Simple but effective passivation process for the mirror facets of high-power semiconductor diode lasers," in Proc. Conf. Lasers Electro-Opt. Eur. Jun. 2003, p. 145 .

[13] M. Farzaneh et al., "CCD-based thermoreflectance microscopy: Principles and applications," J. Phys. D, Appl. Phys., vol. 42, no. 14, p. 143001, Jun. 2009.

[14] D. Pierścińska, "Thermoreflectance spectroscopy-Analysis of thermal processes in semiconductor lasers," J. Phys. D, Appl. Phys., vol. 51, no. 1 , p. 013001,2018

[15] P. W. Epperlein, G. L. Bona, and P. Roentgen, "Local mirror temperatures of red-emitting (Al)GaInP quantum-well laser diodes by Raman scattering and reflectance modulation measurements," Appl. Phys. Lett., vol. 60, no. 6, pp. 680-682, Feb. 1992.

[16] P. K. L. Chan, K. P. Pipe, J. J. Plant, R. B. Swint, and P. W. Juodawlkis, "Temperature mapping and thermal lensing in large-mode, high-power laser diodes," Appl. Phys. Lett., vol. 89, no. 20, p. 201110, Nov. 2006.

[17] P. M. Mayer, D. Lüerßen, R. J. Ram, and J. A. Hudgings, "Theoretical and experimental investigation of the thermal resolution and dynamic range of CCD-based thermoreflectance imaging," J. Opt. Soc. Amer. A, Opt. Image Sci., vol. 24, no. 4, p. 1156-1163, Mar. 2007.

[18] D. C. Hall, L. Goldberg, and D. Mehuys, "Technique for lateral temperature profiling in optoelectronic devices using a photoluminescence microprobe," Appl. Phys. Lett., vol. 61, no. 4, pp. 384-386, May 1992.

[19] S. Arslan, A. Demir, S. Şahin, and A. Aydınlı, "Conservation of quantum efficiency in quantum well intermixing by stress engineering with dielectric bilayers," Semicond. Sci. Technol., vol. 33, no. 2, p. 025001, Feb. 2018

[20] P.-W. Epperlein, "Micro-temperature measurements on semiconductor laser mirrors by reflectance modulation: A newly developed technique for laser characterization," Japanese J. Appl. Phys., vol. 32, no. 12A, pp. 5514-5522, Dec. 1993.

[21] T. R. Chen, L. E. Eng, Y. H. Zhuang, and A. Yariv, "Experimental determination of transparency current density and estimation of the threshold current of semiconductor quantum well lasers," Appl. Phys. Lett., vol. 56, no. 11, pp. 1002-1004, Mar. 1990. 